\title{
Modeling and Presentation of Interdependencies between Key Performance Indicators for Visual Analysis Support
}

\author{
Stefan Hesse, Volodymyr Vasyutynskyy, Martin Rosjat, and Christian Hengstler \\ SAP Next Business and Technology Dresden, SAP AG \\ Chemnitzer Str. 48, D-01187 Dresden, Germany \\ \{s.hesse, volodymyr.vasyutynskyy, martin.rosjat, \\ christian.hengstler\} @sap.com
}

\begin{abstract}
In this paper we propose the modeling and visual presentation of dependencies between key performance indicators. By doing this, explicit presentation and analysis of expert knowledge about dependencies between manufacturing processes can be achieved. This enables route-cause analysis and decision support. Further, using multiple techniques for the visual support we provide the user a high user experience, resulting in a quicker analysis and better user acceptance.
\end{abstract}

Keywords: decision support, visual analysis, key performance indicators, user experience.

\section{Introduction}

Nowadays, key performance indicators (KPIs) are an important instrument to analyze manufacturing systems. They allow the responsible experts or assigned staff e.g. at the production line to get quickly an overview and status of the production processes, detect possible issues, set targets and trigger actions. Accordingly, a lot of software tools support the analysis of KPIs by providing dashboards indicating the status of KPIs etc. Typical dashboards are shown in [1] [2] and [3].

However, these tools are currently limited to a mere visualization of the KPI values. Dashboards lack in scalability for displaying all data. At the same time the processes in modern manufacturing are getting more complex and are tightly connected with each other, so that the processes and their KPIs cannot be considered separately. The analysis of the internal interdependencies between KPIs, which is necessary to assess the causes of possible issues and to make the correct decisions, is still not supported enough by the tools and depends a lot on the experience of human experts. This exposes a lot of potential risks to companies, which include making false decisions due to disregarding of important interdependencies, possible loss of expert knowledge due to human factors etc. Accordingly, this gap should be closed by providing the means for explicit modeling, visualization and analysis of interdependencies between KPIs. 
This paper describes the approach for visual support to analyze KPIs by providing a graph of KPIs showing their status and interdependencies, extended by several features to facilitate the visual perception of the users. The approach is a result of a joint project of several partners towards creating the production logistics and sustainability cockpit (PLANTCockpit, [4]). The approach has been prototypically implemented and tested on a use case from the automotive industry.

\section{Motivation}

In recent years the gathering, interpretation and analysis of business data has become an important key success factor. Single interpreted and calculated values over multiple information items are called key performance indicator (KPI). These indicators describe the state or value of a dedicated object in the business hierarchy. Examples for such indicators are the efficiency of a supplier, duration of the creation of a dedicated product, an error of a machine or the stop of a production line. The key challenges for managers are to have a consolidated and visual fast way of getting an overview about their responsible factory areas as well as potential problems and their causes. Nowadays, KPIs are analyzed using different business intelligence tools like SAP's Business Objects [1] [2]. BI tools can create reports based on data cubes e.g. from data warehouses. Typical reports of these multidimensional items are provided as two dimensional tables, charts or gauges and value based dashboards. Dashboards for KPIs show mostly single values and independent KPIs. As long as more values are stored in a KPI analysis table, these tables may become very complex and need a lot of explanations or background knowledge to be interpreted. This means that after a critical amount of KPIs is reached, those visualizations as tables and dashboards lose their comprehensibility and the semantics of KPIs are not further recognizable anymore. Also interdependencies between different KPIs (in this case cells of tables) are not visual recognizable by the expert within these tables. In most cases these interdependencies are not explicit maintained or even do not exist in common analysis tools, so that the relations between the KPIs (i.e. how they influence each other) are only known from human expert experience or generated by multivariate statistics methods. Since the KPI queries are being created during long periods of time and by different experts, the background knowledge about the interdependencies can get lost over time. If no relations between KPIs explicitly exist, no visual links between the KPIs can be provided automatically.

A typical scenario of KPI analysis is when the cause of the state change of a KPI has to be investigated. For example if the change of a KPI (e.g. KPI A) is influencing another KPI (e.g. KPI B), the cause for changing KPI B can now only be found by background knowledge of the users, but not by direct visibility. This lacking of explicit visualization of logical and visual dependencies makes especially the route cause analysis very hard or impossible. As result, quick and profound decisions cannot be made. Our approach is intended to close this gap. 


\section{Related Work}

Different key performance indicators have been defined in literature and applied in the practice, acquiring quantitative, measurable information and representing them as result of relations and facts [5] [6]. The further development of KPIs and KPI systems into a performance indicator reference model can be found in [7]. The application areas of KPIs include visibility of changes, measurement of current state, comparison of targets and actual business results, decision support, support for coordination and adequate presentation of the information [8] [9] and [10]. Dedicated recommendations for visualization of KPIs can be found in [11].

An investigation of different kinds of data visualization for expert support started in 1960s with the work of Jacques Bertin und Edward Tufte [12] [13]. Also approaches of graph theory for presentation of large data sets appeared [14] [15] [16] [17]. As for now, graphs are a part of different analysis tools as well as visualization libraries. Graphs are also used for different purposes and analysis tools like decision trees, data structures, semantics (RDF), concepts etc. However, till now no approaches are known that support the analysis of KPIs from manufacturing processes using graphbased presentations. Moreover, the approach presented here also includes the method for creation of such KPI dependency graphs. Additionally different interaction features supporting the analysis are introduced.

\section{Graph Model}

\subsection{Graph-Based Presentation of KPIs}

We propose to introduce the graph-based presentation for KPIs to allow the explicit modeling and display of interdependencies. The interdependencies represent the mutual influences between KPIs, and a graph is an intuitive form for representation of such dependencies. Unexpected or undesired KPI state changes can be easily detected. In this way different kinds of analysis will be supported, including root-cause analysis of issues, what-if analysis of future KPI values as well as detecting of possible risks. The elements of the graph-based presentation are shown in Fig. 1.

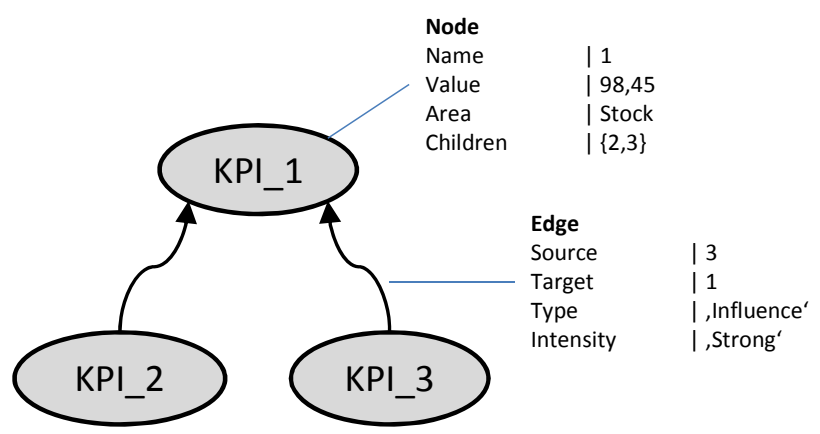

Fig. 1. Elements of the graph-based KPI presentation 
The nodes of the graph represent the KPIs itself. The edges between nodes present the dependencies. Both nodes and edges can have further attributes to represent additional KPI properties, e.g., values, status, etc.

\subsection{Lifecycle of the Graph-Based Presentation}

The realization of the proposed graph-based presentation consists of 2 main phases, a design and a runtime phase. Both phases include further steps which are described in the following.

\section{Design-phase}

In the design phase the configuration of the tool and modeling of dependencies is done. This includes the definition of an overall dependency graph as well as the definition of different user views on the graph created before. First of all abstract KPIs acting as templates for concrete KPI instances are created. Following the dependencies between those abstract KPI templates are modeled. These templates represent generic KPIs without concrete instance relevant properties, e.g., connections to data sources in the business data warehouse. In this state, the design phase can use KPI hierarchies, reference models or results of multivariate statistics methods as input for the creation of the model.

The next step covers the definition of user views. User views contain KPI instances derived from abstract KPIs, e.g., throughput of machine A, which are of interest for the user analysis. This step includes the refinement of the generic KPI properties with concrete values, e.g., the assignment of a query string to a data source. Using the dependencies modeled earlier between abstract KPIs eases the configuration in terms of modeling the dependencies twice or more times on instance level. Detailed information about the different abstraction levels to implement KPIs can be found in [7].

\section{Runtime-phase}

This phase covers the presentation of user views with KPI instances and their values, states, etc. to the user. Depending on the type of analysis (status check, problem cause analysis, what-if analysis etc.), the user interacts with the dependency graph and visually inspects the KPI properties and their status.

\subsection{Features for Support of Visual Perception and User Experience}

To support the visual perception and the user experience, the graph is enhanced with the following interaction features:

- Drill-down possibilities. By clicking on a node, an analysis on its further properties can be made. Folding and expanding of nodes allow the display of a large set of nodes at the same time as well as root cause analysis by expanding and inspecting the relations between single nodes.

- Definition of different user views on top of the overall KPI dependency graph showing different sets of nodes and edges depending on the analysis task and user roles (like manager or dispatcher). For example, the manager is 
interested only in high-level KPIs like overall efficiency or quality ratio. For a dispatcher, the status of the production line (e.g. throughput of the production line) and single machines (working/not working) is important. By choosing the corresponding view, the user can immediately see only the KPIs and dependencies he is interested in and authorized for.

- Definition of sub-graphs and groups of nodes representing specific areas of interest. The sub-graphs or groups can be again folded to limit the visual overload for the user when viewing large graphs.

- Drag-and-drop of single nodes, node groups or sub-graphs to support easy creation of the KPI dependency graph as well as user views.

- Highlighting of nodes or edges, e.g., based on values. For example, the color of a node may represent if the KPI values is within acceptable limits defined by matching targets (e.g., green) or exceeds these limits, (e.g., yellow or red).

- Filtering (e.g. hiding or increasing opacity) of nodes according to different criteria. During the interactive analysis, the user may be interested only in a subset of KPI instances, which is supported by filtering. For example by setting the criteria "area=Painting", the user will see only KPIs from this area.

- Graph layout algorithms like Sugiyama or energy-based [18] [19] can be used to adjust the presentation to the users' expectations. For example, for hierarchical KPIs should be intuitively presented by hierarchical trees.

\section{Implementation}

The proposed concepts are implemented in a prototype shown in Fig. 2 and developed in .Net. The prototype retrieves the KPI data and values from a connected business warehouse system out of test OLAP cube. The prototype has been applied on sample data of a use case from automotive industry and presents an exemplified user view.

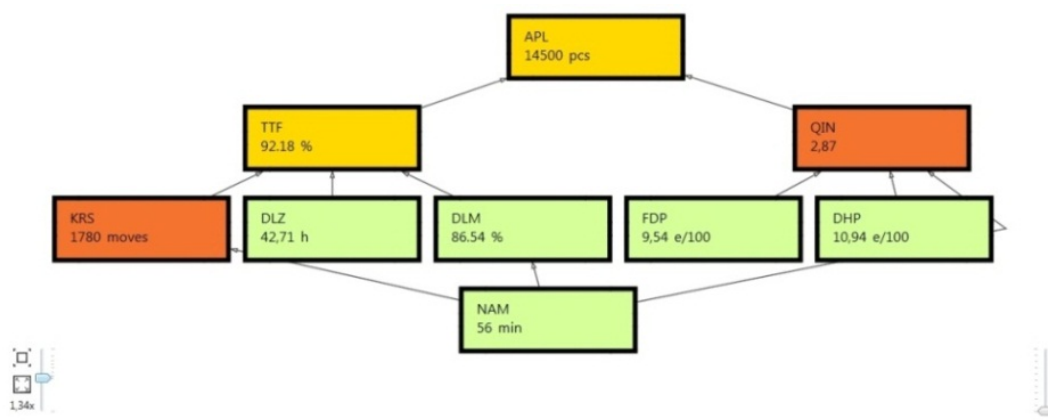

Fig. 2. Sample user view of a KPI dependency graph of the prototype. States of KPIs are represented by different colors of nodes.

The implementation is based on the PLANTCockpit architecture described in [20]. This component based architecture is designed for gathering, interpreting and analyz- 
ing data from heterogeneous data sources such as ERP, MES, Files, databases and data warehouses or other external systems.

The prototype implements the user experience features of graph visualization described in Section 4.3. Using the paradigm of Zoomable User Interfaces (ZUI), the expert can dive into large graphs with up to thousands of KPIs (see Fig. 3), still seeing most relevant parts.

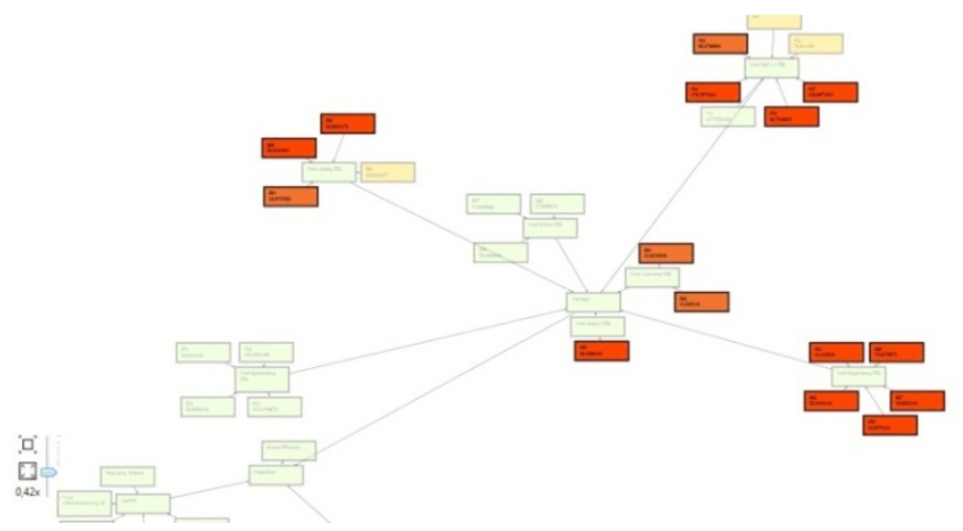

Fig. 3. Filtering support on different KPI states, names or tags

According to user's preferences large graphs, sub-graphs or groups of KPIs can be folded, expanded or repositioned by the user. Furthermore, the prototype allows the filtering of nodes using content, tags or states of the single KPI. In our prototype, the result of the filtering is shown in a decrease of the opacity of non-relevant KPIs. The filtering on the status of KPIs is depicted in Fig. 4. The automatic use of different graph layout algorithm allows the creation of KPI representations according to the user's expectations.
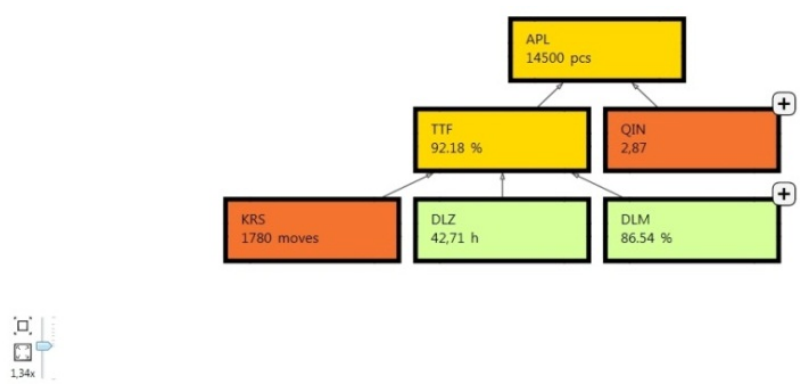

Fig. 4. User support by folding of sub graphs

The prototype was tested on the use cases of KPI analysis of manufacturing KPIs of a large automotive company. The prototype was connected to the data warehouse of the company, allowing presentation of the status of the real KPIs. The data 
warehouse contains thousands of KPI types as well as their current and historical values, which are usually accessed by data cube queries, based on which the table- and chart-based reports are created.

The KPI graphs have been created together with the experts of the company. Using our approach, the analysis of the huge amounts of KPIs could be managed in an easier way, facilitating and accelerating the work of human experts.

\section{Conclusion and Future Work}

The proposed approach will enhance the decision support by a higher visibility of respective influences and dependencies. It allows root-cause and what-if analysis for KPIs by utilizing links between dependent KPIs. With this capability the approach can be used for analysis of any complex business process, among others in the production or logistic management, and for production performance measurement.

With the presented method, the implicit logical connections between KPIs are turned into visual dependencies. This allows the user to explore KPIs and their dependencies in a visual way. Using a graph for visualization and interpreting KPI data allows a much more interactive presentation than tables and reports. This interactive presentation is enhanced with visual support like filtering, drag and drop or zoom.

One of our future works will cover further enhancements in interactivity and a user test with KPI experts from the automotive industry to refine the assumed models and to enhance the user experience. This test will lead to new insights into the interpretation and realization of KPI results using our visual approach. An additional study will provide information about the effects of the approach for our use case partner.

Acknowledgment. The authors would like to thank the European Commission for co-funding of the research project PLANTCockpit (under the project number 260018).

\section{References}

1. SAP AG: Simple, Affordable, and Open BI Tools for Everyday Use. In: SAP Crystal Solutions, http: / / www.sap.com/solutions / sap-crystal-solutions/index.epx

2. SAP AG: See Your Business Clearly. In: SAP BusinessObjects Dashboards, http: / /www.sap.com/uk/solutions/sapbusinessobjects / large/business-intelligence/dashboards/ sapbusinessobjects-dashboards/index. epx

3. Siemens AG: SPPA-M3000 Plant Management. In: Siemens, http: / /www. energy. siemens.com/co/en/automation/ power-generation/sppa-m3000/sppa-m3000-plantmanagement .htm\#content=Description

4. The PLANTCockpit Consortium: PLANTCockpit EU-funded Project, http: / /www.plantcockpit.eu 
5. International Organization for Standardization (ISO): ISO/CD 22400-2: Automation systems and integration - Key performance indicators (KPIs) for manufacturing operations management - Part 2: Definitions and descriptions (May 2012)

6. Reichmann, T.: Controlling mit Kennzahlen und Management-Tools: Die systemgestützte Controlling-Konzeption, 7th edn. Vahlen, München (2006)

7. Hesse, S., Wolf, B., Nadoveza, D., Pintzos, G., Rosjat, M.: Reference model concept for structuring and representing performance indicators in manufacturing. In: Emmanouilidis, C., Taisch, M., Kiritsis, D. (eds.) APMS 2012, Part II. IFIP AICT, vol. 398, pp. 289-296. Springer, Heidelberg (2013)

8. Gladen, W.: Kennzahlen- und Berichtssysteme.: Grundlagen zum Performance Measurement. Gabler (2001)

9. SAP AG: SAP Developers Network - KPIs, http://wiki.sdn.sap.com/wiki/display/KPI

10. Chryssolouris, G.: Manufacturing Systems: Theory and Practice, 2nd edn. Springer (2006)

11. Wenzel, S., Bernhard, J., Jessen, U.: Visualization for modeling and simulation: a taxonomy of visualization techniques for simulation in production and logistics. In: Proceedings of the 35th Conference on Winter Simulation: Driving Innovation, pp. 729-736 (2003)

12. Bertin, J.: Semiology of graphics. University of Wisconsin Press (1983)

13. Tufte, E.: The visual display of quantitative information. Graphics Press (1986)

14. Lamping, J., Rao, R., Pirolli, P.: A focus+context technique based on hyperbolic geometry for visualizing large hierarchies, pp. 401-408 (1995)

15. Wattenberg, M.: Visual exploration of multivariate graphs, pp. 811-819 (2006)

16. Horsfall, F., Tanev, S., Bontchev, B., Gigilev, T., Gruev, A.: Visualization of complex data relationships and maps: using the BLOOM platform to provide business insights, pp. 266-272 (2011)

17. Doganata, Y., Topkara, M.: Visualizing meetings as a graph for more accessible meeting artifacts, pp. 1939-1944 (2011)

18. Eiglsperger, S.: An Efficient Implementation of Sugiyama's Algorithm for Layered Graph Drawing (2005)

19. Noack, A.: An Energy Model for Visual Graph Clustering (2006)

20. Vasyutynskyy, H.C., Nadoveza, D., McCarthy, J., Brennan, K.G., Dennert, A.: Layered Architecture for Production and Logistics Cockpits. In: 17th IEEE International Conference on Emerging Techonologies and Factory Automation (ETFA), Krakow, Poland (2012) 